\title{
OPERATION FOR MAKING THE ARTIFICIAL VAGINA
}

\author{
(NAKAYAMA'S METHOD) \\ YASUSHI NAKAYAMA \\ Department of Gynecology, School of Medicine \\ Keio University
}

(Received on December 6, 1956)

This surgical procedure is performed in case of congenital lack of the vagina or severe closing of it. On ordinary closure of the reproductive canal, such as partial closure of the vagina or the imperforated hymen, no reference will be made here.

Now, there are some important differences, between the way of operation which I am going to describe and Schubert's method, though the idea may be common between them, to use a part of the rectum as the artificial vagina. I have named my way of operation as Nakayama's method.

\section{HISTORY OF THE OPERATION}

Posibility of making the artificial vagina was suggested by the operation which was known as a treatment for hematoma uteri, in which a trocar was inserted between the bladder and the rectum to remove the accumulated blood. An attempt was made, at the begining, to give a sharp dessection there, instead of inserting a trocar. But the results were unsuccessful and mostly they became lifeless from acute septicemia. Amussat (1832) marked a brilliant step in the developement of this kind of operation by suggesting a blunt dessection and thus eliminating sharp trauma to the tissue and making hemorrhage minimal. It soon became apparent, however, the new disected tract by his way of operation would eventually contract owing to cicatrization. Here arose the important problem how to prevent its contraction for life time and thus to preserve its sexual function of the newly formed vaginal space. Many attempts were made, then, to cover the newly formed tract with epithelium. But the technique, itself, to cover the tract was much difficult and morevover the transplanted epithelium became necrotic, in most cases. Nevertheless, a number of trials were performed to transplant the skin from the labia major, the labia minor or the perineum in the form of a tube to cover the inside of the newly formed tract. It is true 
that there are a few successful reports by these methods, but I am much doubtful whether their results were good enough to have had attained their expected purpose, the complete prevention of the contraction owing to cicatrization. As wider coverings, they tried the mucosa of the rabbit's gut, the skin from the hip, the peritoneum or even the vaginal mucosa from an operated case of prolapsus uteri, but the results would be much worse.

\section{METHODS USING A PART OF INTESTINES}

As stated above, the types of operation which use skin grafts of flaps to cover the newly formed vaginal space, were unsatisfactory and they hardly worth to be paid any attention nowadays. The method which is considered most reasonable today is the one which uses a part of intestines to form the artificial vagina. In this method, there are two types of operation, one of which is to utilize a part of the small intestine and the other use a part of the rectum.

1) Gersuny's (Rectum) Method (1897)

A skin petal like flap of the mucous membrane of the vaginal entrance is used to form the anterior wall of the artificial vaginal space which is made between the bladder and the rectum. The posterior wall is formed by a portion of the rectum.

2) Mori's (Journ. of Japan Gynecolagical Ass. 3rd issue, 3rd year, 1908) and Baldwin's (Journ. of the Ameri. Med. Associ. Nr. 111, 1910) (Small Intestine) Method

A resected portion of the small intestine is utilized in the opening made between the bladder and the rectum. In Mori's method, the small intestine is used lengthwise whereas in Baldwin's method, it is used in the shape of a horseshoe. It will be agreeable to call this method, Mori's and Baldwin's as Dr. Kakuichi Ando has suggested. Each of them had created the method without any knowledge of the other's.

3) Schubert's (Rectum) Method (Centralbl. f. Gyn. Nr. 28, 1911)

The amputated part of the lower rectum is transferred to the normal position of the vagina and forms the artificial vagina. The edge of the upper portion of the rectum is pulled downwards to the anal opening and there sutured to the anal sphincter.

No laparotomy is required in this method.

\section{COMMENT ON THE PREVIOUS METHODS}

There is no doubt today that contraction owing to cicatrization is unavoidable 
for any method which cover with skin grafts or flaps the inside of the vaginal space. What is the use, then, of the kind of operation which is so hard and so laborious to perform when the new tract as the vagina shrinks eventually and can not maintain for life time its sexual function. I am not able to understand why these classical ways of transplantation method are still being used even at present. I can not but consider it is performed for temporal and sentimental comforts of the patient. Of late, a successful epithelization on the newly dissected vaginal space is reported. Although, there may be some academic importance in it, it is no use from the practical point of view, if not the phenomenon means the successful prevention of contraction. I, therefore, emphasise that these transplantation methods are not worthy trying today, and that even the successful epithelization, which will hardly happen, does not contribute to the realization of the practical and durable artificial vagina. Now, the way of operation which is to be reasonably recommended from the standpoint of the up-to-date clinical surgery is the intestine method.

Among them, the small intestine method such as Mori' and Baldwin's, is not exempted from the fundamental deficiency. There may be some cases successfully reporting the prevention of contraction by this small intestine method but it is much questionable if it last for life time. Then, the only way remained for wide acknowledgement is the rectum method. There are three methods of this kind, that is Gersuny's, Schubert's and my Nakayama's method belongs to this type, too. Gersuny's method is a part transplantation method and is to be excluded. Among the various surgical procedures previously introduced in this field, Schubert's method succeeded, for the first time, in the perfect prevention of contraction owing to cicatrization. But, to our regret, his method suffers another important faults. First of all, the separation of the rectum by his method requires much difficult and complicated process and so exessive bleeding is inevitable and consequently the results become unsatisfactory. Moreover, the rectum which is isolated by such difficult undertaking frequently shows to be not having enough length for the new rectum after dessecting the required portion at first for the new artificial vagina. The new rectum not having proper length will pull inwards the sutured region of the anus and finally results in separation of the sutures and formation of scar there.

\section{MY WAY OF OPERATION}

Preparation:

1. Use a purgative at the previous night.

2. On the day, let the patient fast, and give a soap enema. 
3. Just before the operation, wash the rectum carefully with plenty of 2\% tepid boric acid solution. Pay special attention to exclude the solution completely after washing.

Anesthesia: Lumbar anesthesia is used.

Time: It will take about one hour and fifty minutes.

The System of the Operation:

A. Incision and separation for the new vaginal entrance, and the isolation of mucous membrane of the anus.

B. Laparotomy for the isolation and amputation of the rectum, and pulling down the rectum for the new anus.

C. Making the new vagina and the new anus.

Operation $A$.

1. Incision and separation for the new vagina.

a. Incise horizontally about $3 \mathrm{~cm}$ or about 1 inch. (Fig. 1)

b. Separate not so deeply, not more than $1 \mathrm{~cm}$ or 0.4 inches. (Fig. 2)

2. Isolation of mucous membrane of the anus.

a. Incise circularly the mucous membrane of the anus and isolate the lowest part of the rectum $1 \mathrm{~cm}$ or 0.4 inches in front and $5 \mathrm{~cm}$ or 2 inches in back. (Fig. 3-4)

b. Close the end of the rectum (Fig. 5) and insert a piece of gauze behind the isolated lower part of the rectum. (cf. Fig. 9)

Operation B. Laparotomy

1. Incision of the Peritoneum.

Incise peritoneum along the left and right side of the rectum from the prominency of the sacrum to the Douglas's Pouch. (Fig. 6-7)

2. Isolation of the rectum.

Isolate the rectum by fingers and scissors, if necessary, down to the lower part of the rectum. (Fig. 8-9) The gauze (A.2.b.) will be an important indicator in this process.

This isolation process in Nakayama's method is very simple and easy and bleeding will be little. This is one of the superior points compared with Schubert's method.

3. Amputation of the rectum.

a. As in the case of ordinary intestinal operation, amputate the rectum between intestinal clamps at $11-12 \mathrm{~cm}$ or $4.5-5$ inches from the anus. (Fig. 10)

b. Suture the upperedge of the amputated rectum (Fig. 11) and 
wrap it with gauze.

c. When the clamp is removed, be careful no solution will flow out which was used for washing the rectum before the operation. Before the suture, clean the inside of the rectum with gauze and paint thin iodine tincture there and pack a piece of iodoform gauze in it. (Fig. 12) Then, suture it. (Fig. 13)

4. Pulling down the upper rectum for the new anus.

a. Isolation of the upper rectum.

Isolate the upper portion of the rectum up to the $\mathrm{S}$ shaped colon to obtain enough length for the new rectum. (Fig. 14)

There may be more bleeding than the case of the lower rectum, but it is not necessary of worry about it.

b. Pulling down the rectum.

When the required length of the rectum was made sufficiently movable, seize the gauze wraped edge of the upper rectum (Fig. 15) and pull downwards (Fig. 16) until it will appear at the anal region and behind the former rectum which is to be the artificial vagina. (Fig. 17)

c. Suture of the peritoneum

Suture the peritoneum so as to all the wounds will be set outside of it. (Fig. 18-19)

\section{Operation $C$.}

1. Making the new vaginal entrance.

a. Incision of the rectum.

Incise the rectum which is to be the new vagina through the new vaginal entrance. (Fig. 20-21) Then the iodoform gauze inserted in the rectum (B.3.c.) will be an important indicator.

b. Suture of the rectum.

Suture carefully with cutguts the former end of the rectum.

c. Making the new vaginal entrance.

Suture by knot the edge of the vestibular mucosa to the edge of the incised rectum. (Fig. 22) You can make the new vaginal entrance wide or narrow by incising the rectum much or little. This is one of the strong points of Nakayama's method. There may be some bleeding in this process and a gauze tampon is put there.

2. Fixation and opening of the new anus. 
a. Former anal end of the rectum.

Push inwards the former anal end of the rectum. (Fig. 23)

b. Making the new anus.

Suture the new rectum to the anal sphincter (Fig. 24) and cut off the temporarily sutured end of the rectum. (Fig. 25)

c. Replace the gauze in the new vagina and put a piece of gauze at the new anus.

Now, the new artificial vagina and the new anus have been completed. (Fig. 26-28)

After the Operation:

Stop the evacuation for about 7-10 days. Notice:

Considering my experiences, some changes in the process were made as follows:

a. The incision of the vestibular mucosa (Fig. 1) shall be done after the laparotomy is performed. (The procedure Fig. 1-2) are to be made after Fig. 19)

b. After the sutured former anal end of the rectum (C.2.a.) is pushed inwards (Fig. 23), it is covered by suturing the anterior part of the separated surface of anal region. By such management, fistel is avoided which sometimes occurs between the new vagina and the new rectum. (cf. Fig. 27-28)

\section{NAKAYAMA'S METHOD}

Nakayama's method enjoy, with Schubert's, the virtue that it is free from the fundamental defect of contraction owing to cicatrization which have not been prevented by any other method, by using the rectum as the artificial vagina. Besides, by adopting laparotomy, it has succeeded to make the separation of the rectum so easy and to minimize its hemorrhage. Now, this effectual operation for making the artificial vagina is safe for anyone who can undertake ordinary intestinal operation. And, you shall notice that, by this method, the entrance of the artificial vagina may be made as expected, large or small. For it does not use the anal end of the rectum for new vaginal entrance as in the case by Schubert's method, but the incised edge of the rectum is sutured to the opening in vestibular mucosa. In conclusion, the artificial vagina formed by Nakayama's method will not contract for life time and the procedure of the operation itself does not comply any danger of unsuccessful results. It is especially true when penicillin may be abundantly utilized. 


\section{IT'S HISTORY}

Dr. Shohei Takayama, my skilled professor when I had been in the gynecological department of Kyoto Imperial University, had been operating the case by Gersuny's method at the beginning. Afterwards, he had changed it to Mori's and Baldwin's method, but he had still been unsuccessful. In later days, I have become an assistant professor in Keio University, Medical Department. Then, I had some chances to take part in the operation for making the artificial vagina as by Schubert's method, but the results were also not satisfactory. The fact that these operations done by the most skilled hands still had resulted unsuccessfully indicated me of some fundamental inherent faults in these intestine methods. It was in 1925 when I had performed the first operation in my own method, which result was excellent and was named Nakayama's method. It is my great pleasure to describe here Nakayama's method for making the artificial vagina which function will last for life time and the operation is safe and easy as proved by 30 years of my experiences. 

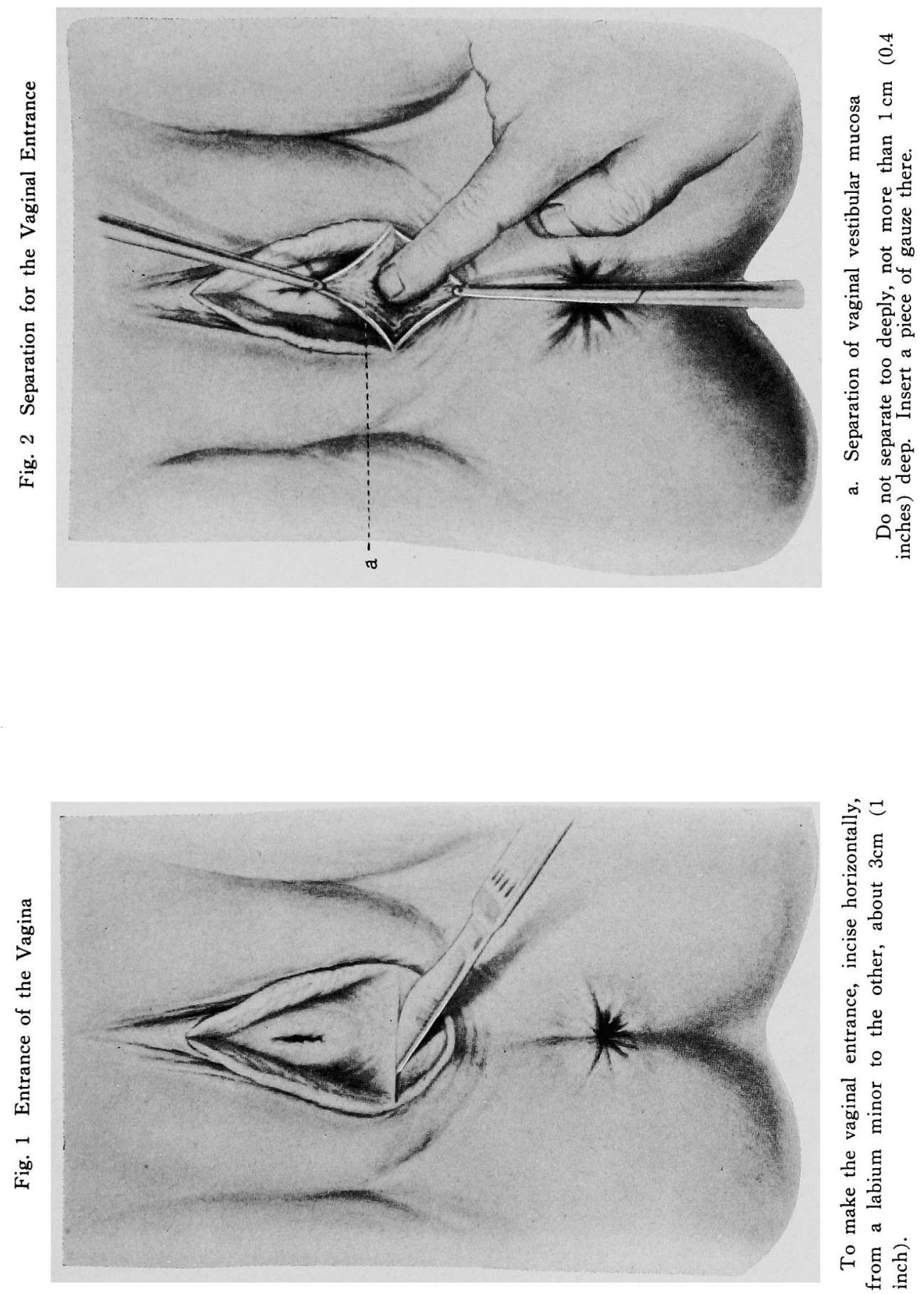

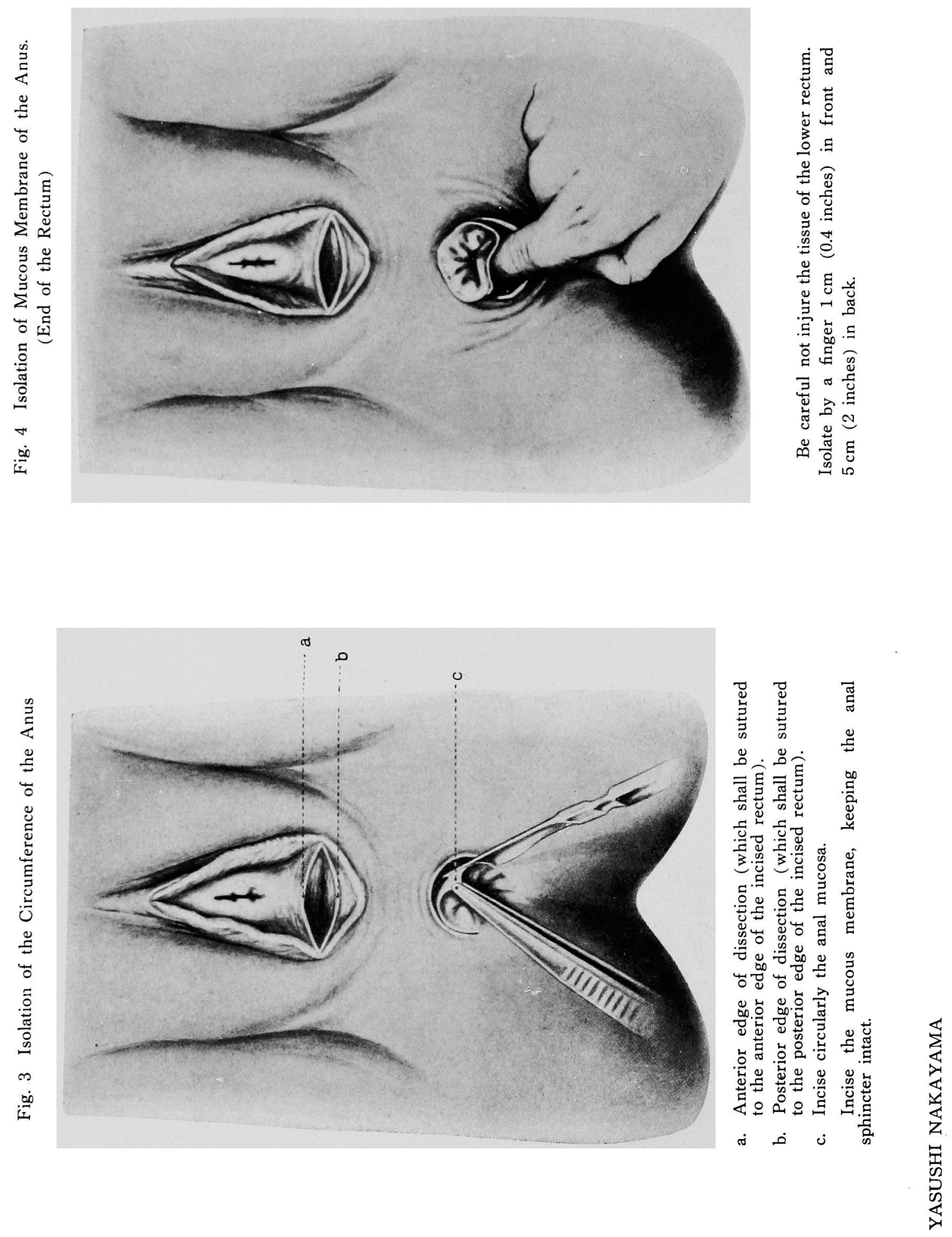

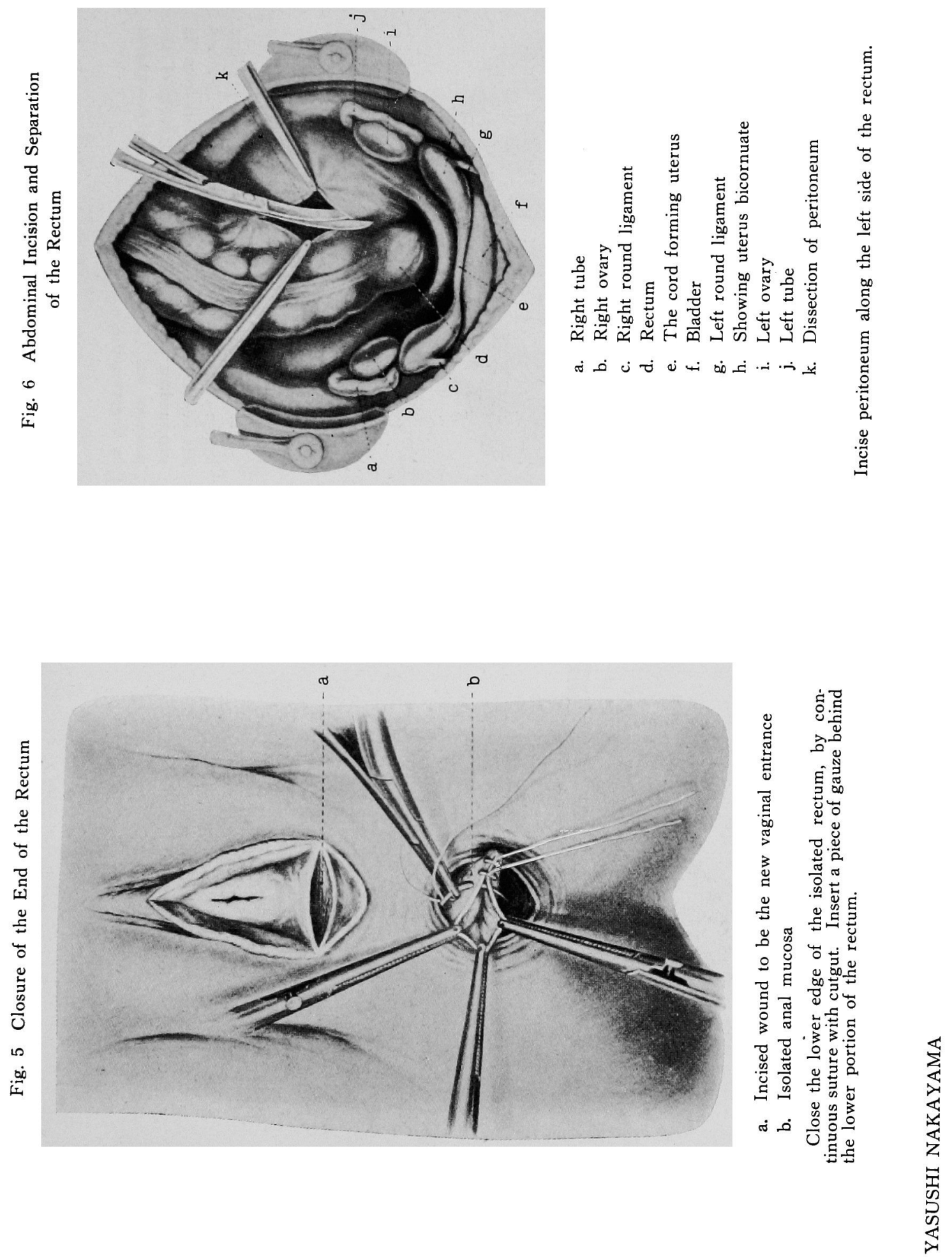

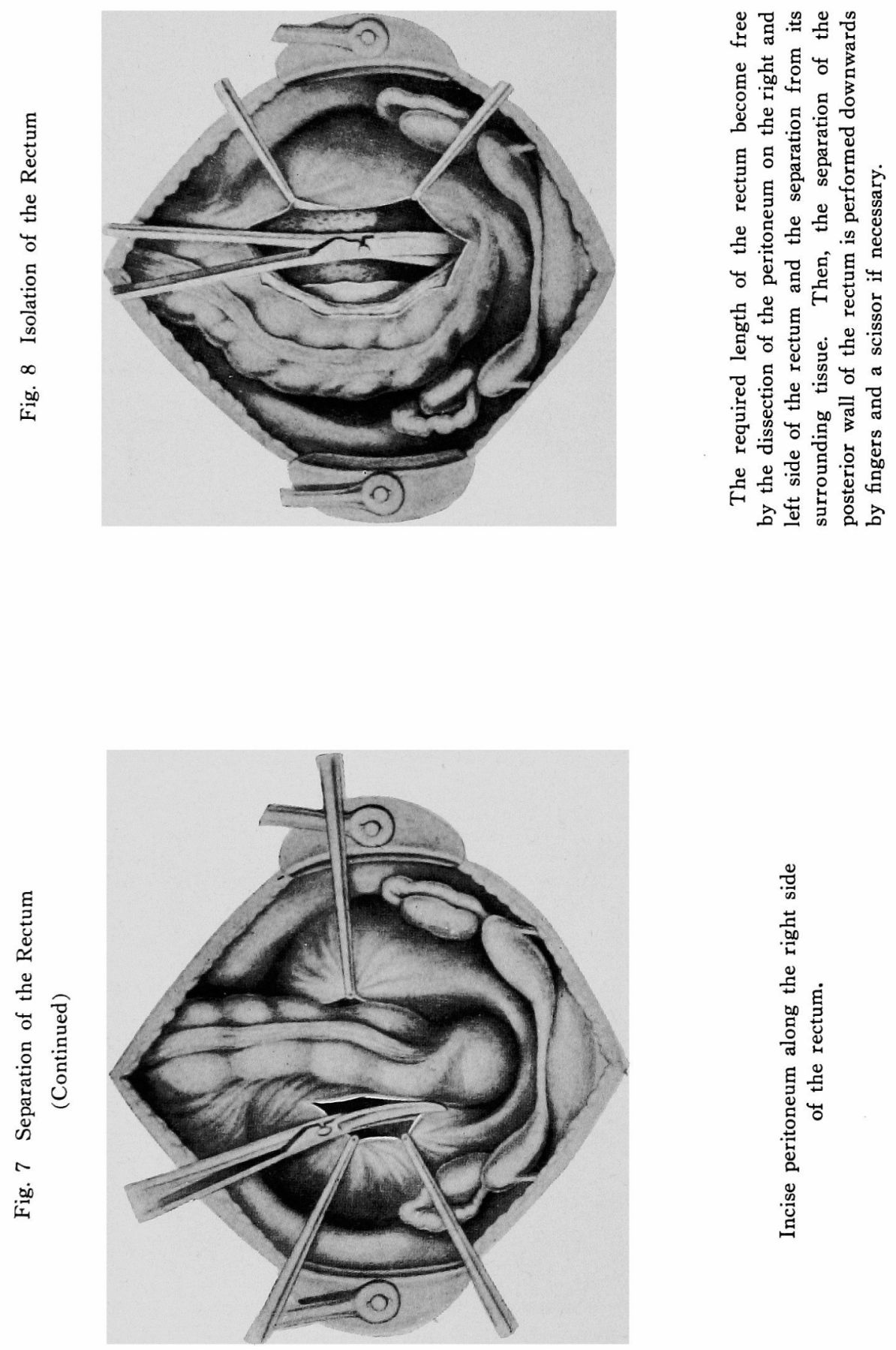



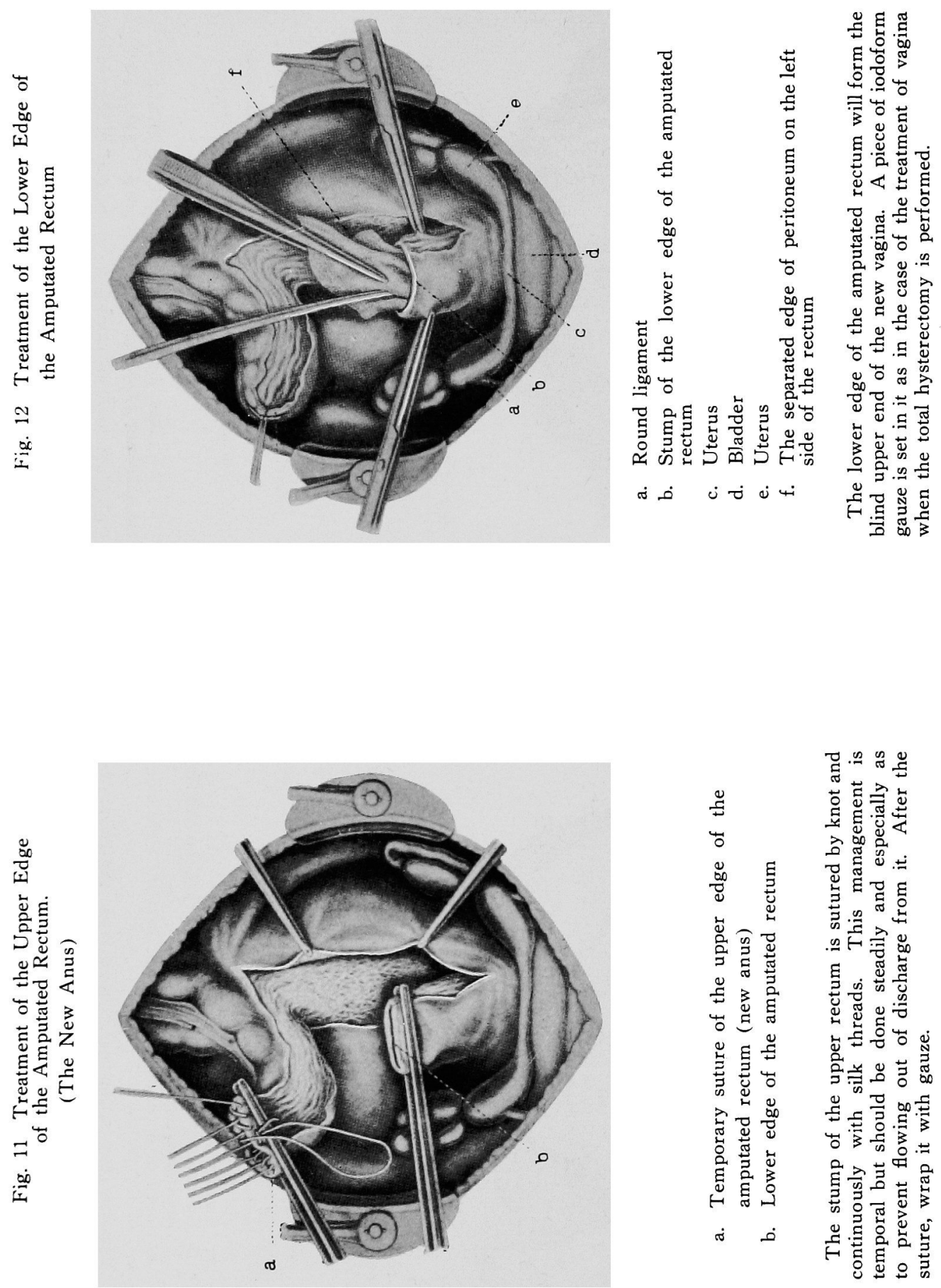

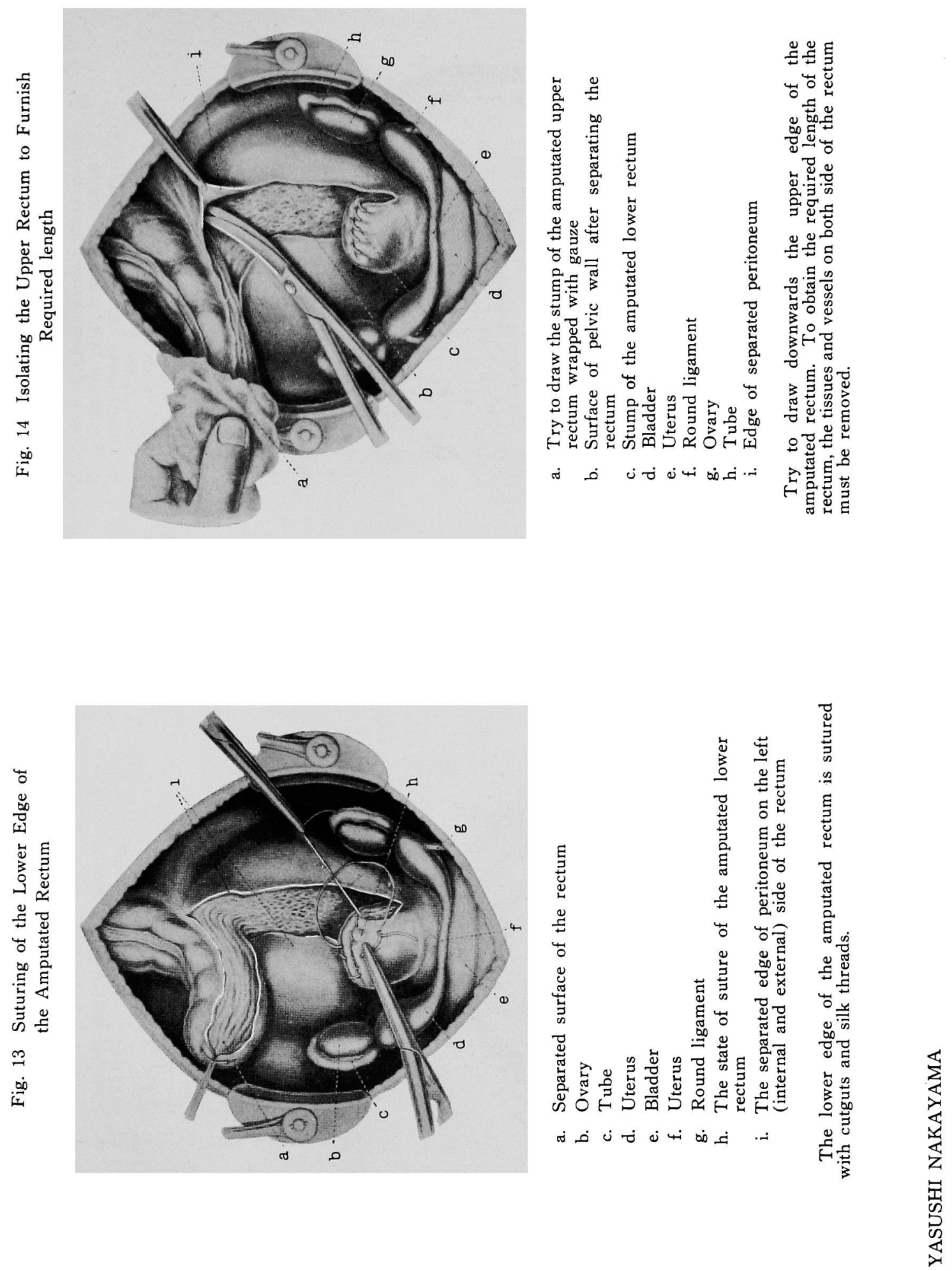

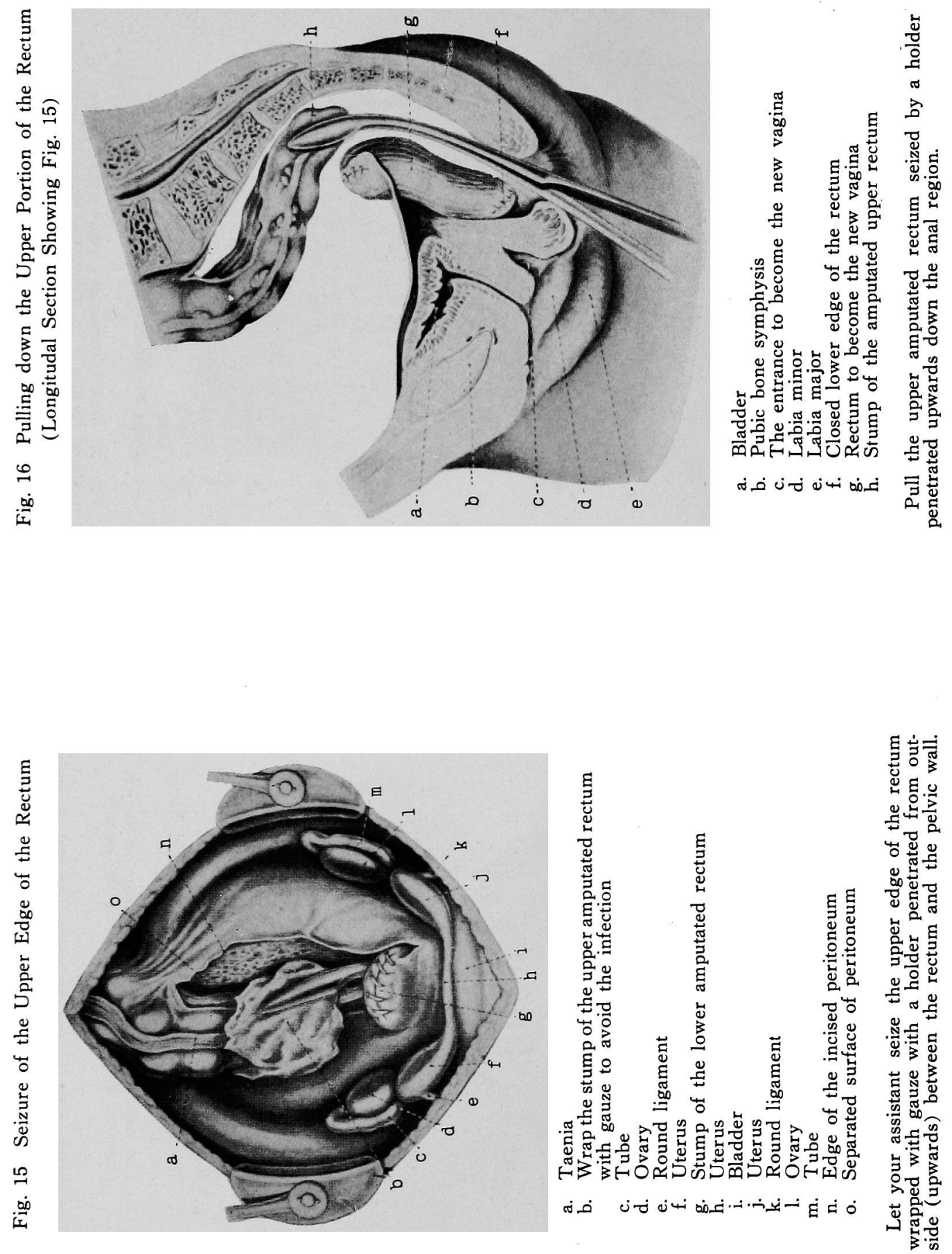

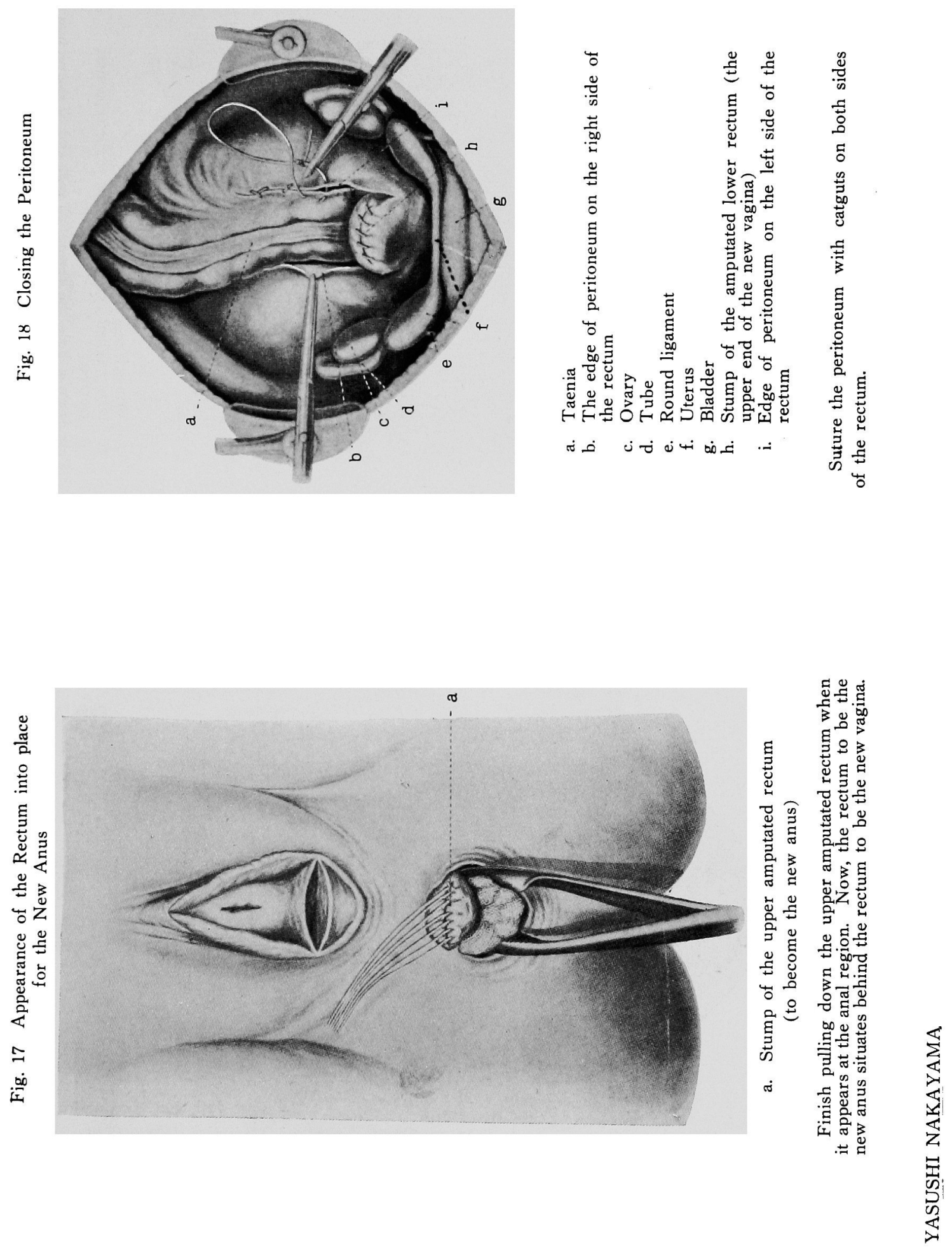

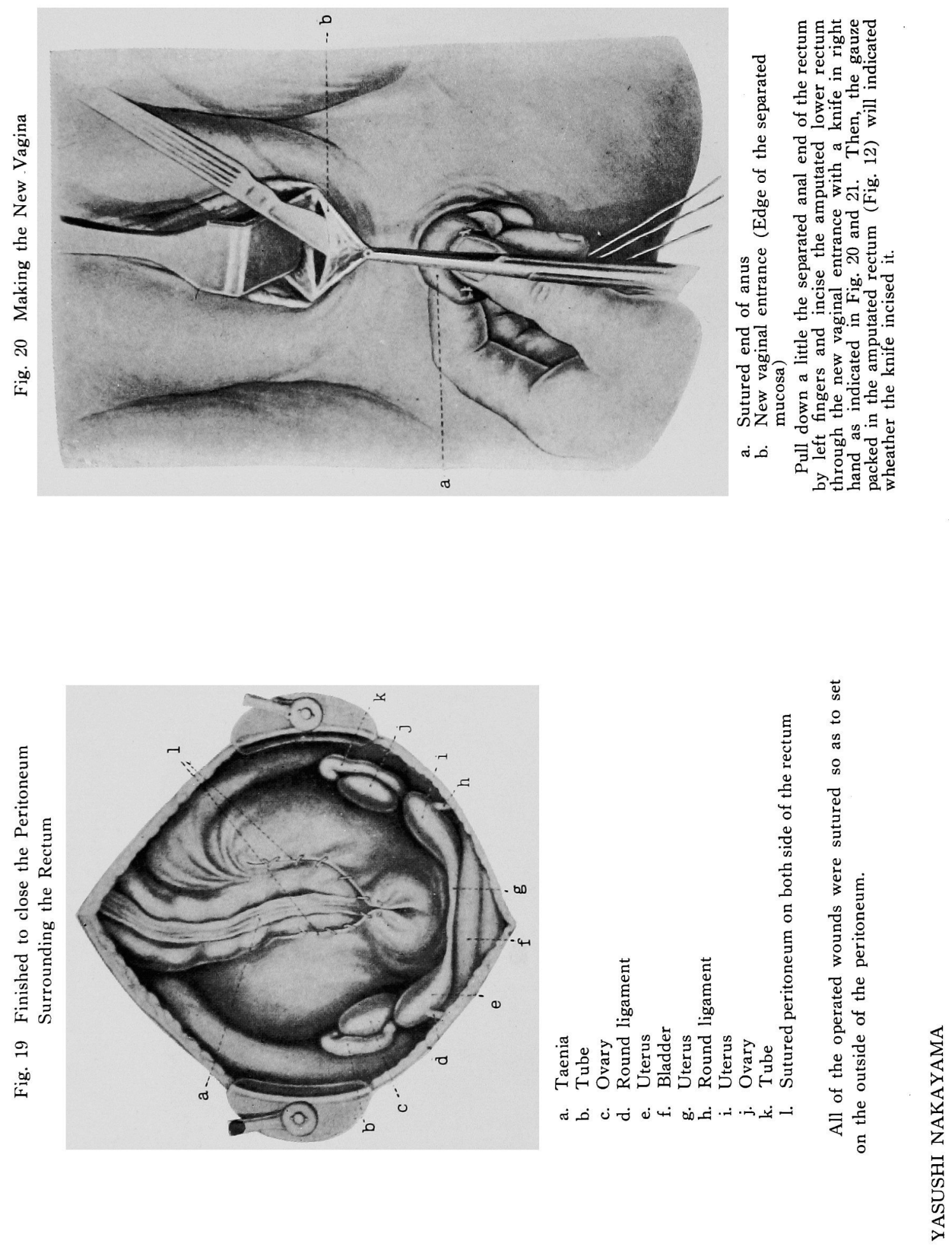

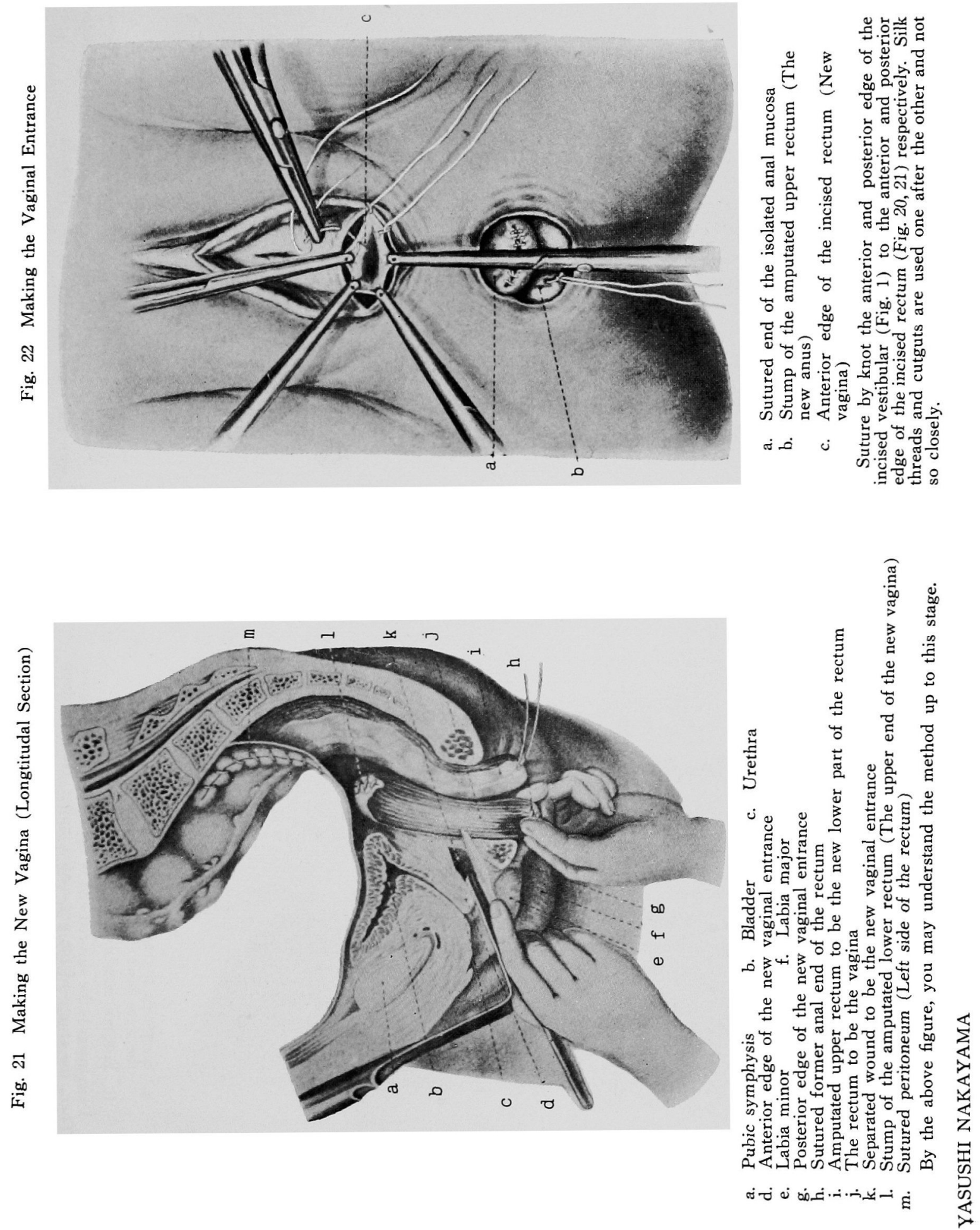

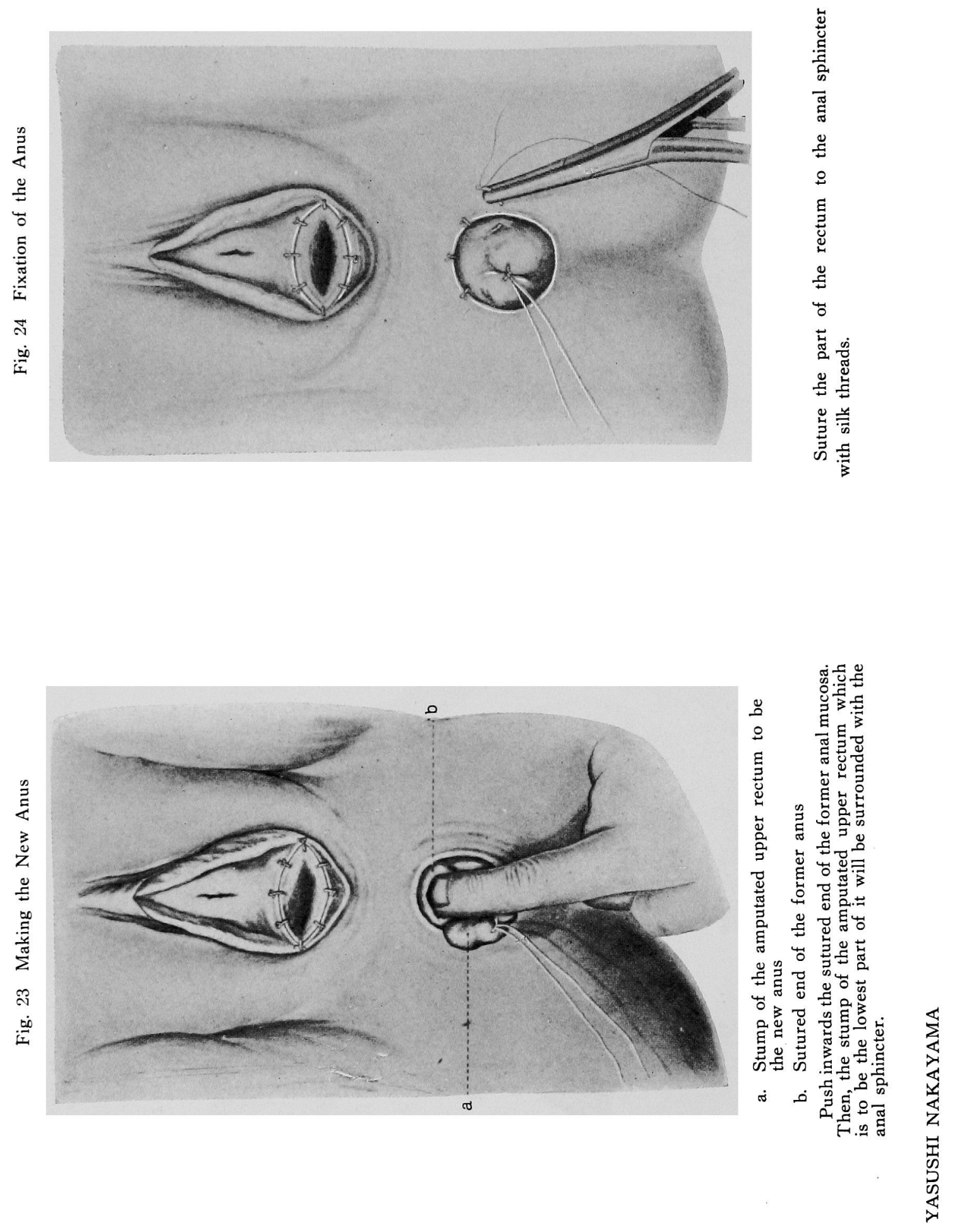

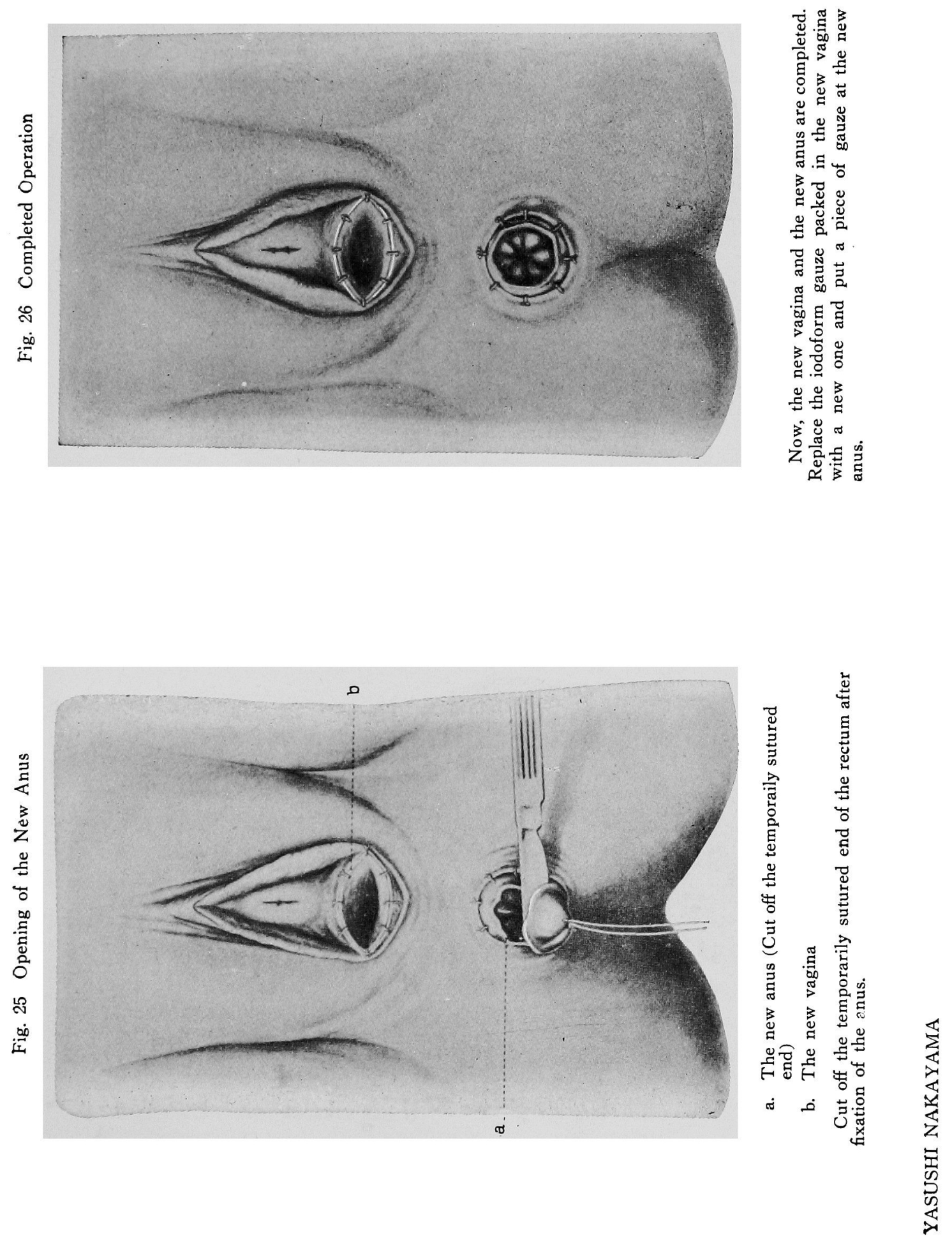

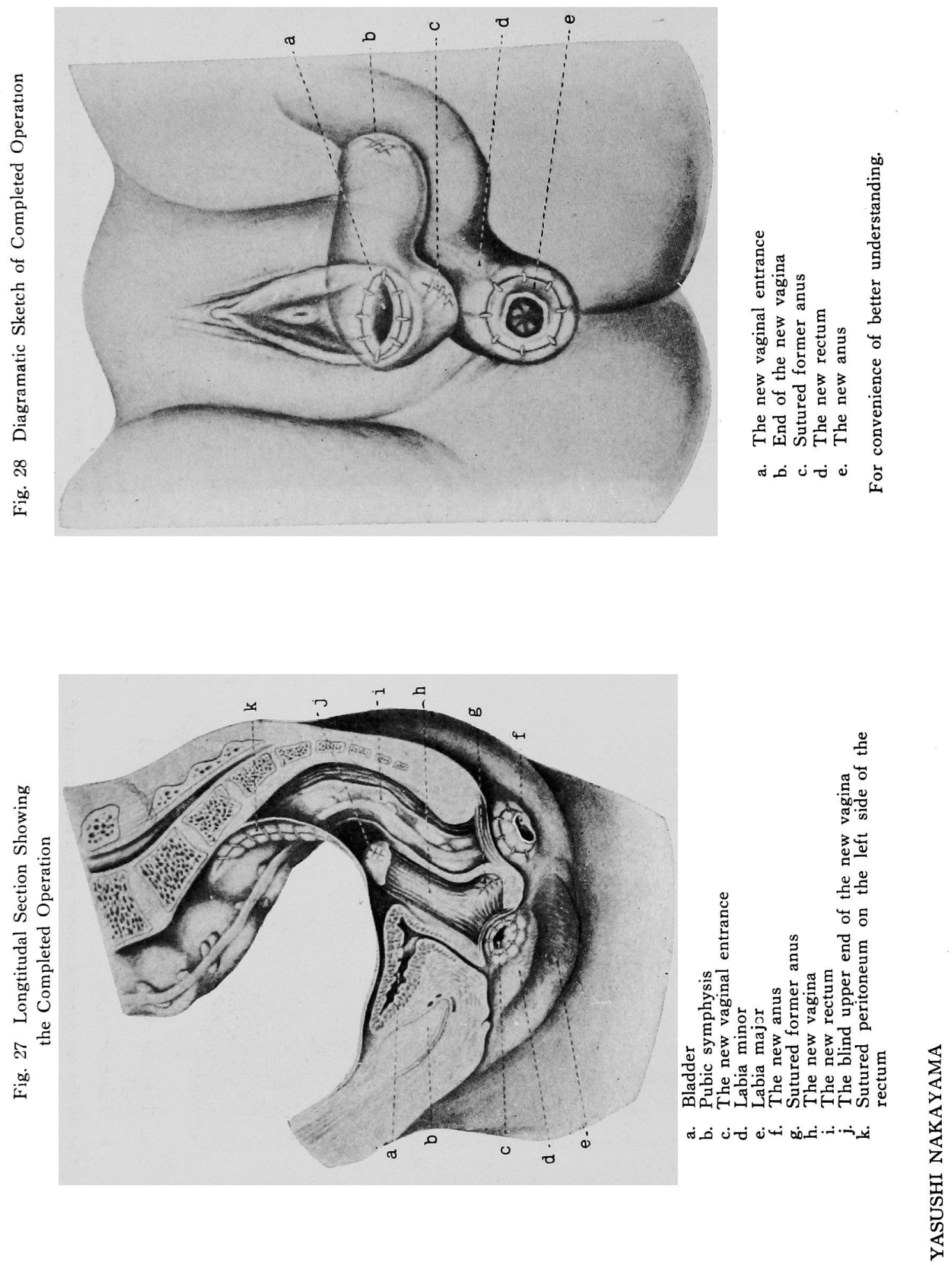